\title{
Pulse Vaccination Strategy in an Epidemic Model with Two Susceptible Subclasses and Time Delay
}

\author{
Youquan Luo, Shujing Gao, Shuixian Yan \\ Key Laboratory of Jiangxi Province for Numerical Simulation and Emulation Techniques, \\ Gannan Normal University, Ganzhou, China \\ E-mail: gaosjmath@126.com \\ Received August 1, 2010; revised November 10, 2010; accepted November 15, 2010
}

\begin{abstract}
In this paper, an impulsive epidemic model with time delay is proposed, which susceptible population is divided into two groups: high risk susceptibles and non-high risk susceptibles. We introduce two thresholds $R_{1}$, $R_{2}$ and demonstrate that the disease will be extinct if $R_{1}<1$ and persistent if $R_{2}>1$. Our results show that larger pulse vaccination rates or a shorter the period of pulsing will lead to the eradication of the disease. The conclusions are confirmed by numerical simulations.
\end{abstract}

Keywords: High Risk Susceptible, Non-High Risk Susceptible, Pulse Vaccination, Extinction

\section{Introduction}

Infectious diseases have tremendous influence on human life. Every year, millions of people die of various infectious diseases. Controlling infectious diseases has been an increasingly complex issue in recent years [1]. Over the last fifty years, many scholars have payed great attention to construct mathematical models to describe the spread of infectious diseases. See the literatures [2-8], the books [9-11] and the references therein. In the classical epidemiological models, a population of total size $N$ is divided into $S$ (susceptible numbers), $I$ (infective numbers), or $S, I$ and $R$ (recovered numbers) or $S, E$ (exposed numbers), $I$ and $R$, and corresponding epidemiological models such as SI, SIS, SIR, SIRS, SIER and SEIRS are constructed. All these models are extensions of the SIR model elaborated by Kermack and McKendrick in 1927 [12]. Anderson and May [5,9] discussed the spreading nature of biological viruses, parasites etc. leading to infectious diseases in human population through several epidemic models. Cooke and Driessche [8] investigated an SEIRS model with the latent period and the immune period. The consideration of the latent period and the immune period gave rise to models with the incorporation of delays and integral equation formulations.

However, owing to the physical health status, age and other factors, susceptibles population show different in- fective to a infectious disease. In this paper, we divide the susceptible population into two groups: nonhigh risk susceptibles $\left(S_{1}\right)$ and high risk susceptibles $\left(S_{2}\right)$, such that individuals in each group have homogeneous susceptibility, but the susceptibilities of individuals from different groups are distinct. In this paper, we propose a new SIR epidemic model, which two noninteracting susceptible subclasses, the nonlinear incidence $\beta S^{p} I$, time delay and pulse vaccination are considered. The main purpose of this paper is to study the dynamical behavior of the model and establish sufficient conditions that the disease will be extinct or not.

The organization of this paper is as follows. In the next section, we construct a delayed and impulsive SIR epidemic model with two noninteracting susceptible subclasses. In Section 3, using the discrete dynamical system determined by the stroboscopic map, we establish sufficient conditions for the global attractivity of infection-free periodic solution. And the sufficient conditions for the permanence of the model are obtained in section 4. Finally, we present some numerical simulations to illustrate our results.

\section{Model Formulation and Preliminaries}

Gao etc. [13] proposed a delayed SIR epidemic model with pulse vaccination: 


$$
\left\{\begin{array}{l}
S^{\prime}(t)=\mu-\beta S(t) I(t)-\mu S(t), \\
I^{\prime}(t)=\beta S(t) I(t)-\beta e^{-\mu \tau} S(t-\tau) I(t-\tau)-\mu I(t), \\
R^{\prime}(t)=\beta e^{-\mu \tau} S(t-\tau) I(t-\tau)-\mu R(t), \\
S\left(t^{+}\right)=(1-\theta) S(t), \\
I\left(t^{+}\right)=I(t), \\
R\left(t^{+}\right)=R(t)+\theta S(t),
\end{array}\right\} t \neq n T, n \in N
$$

In model (1), the authors assumed that the birth rate $(\mu)$ is equal to the death rate, and use a bilinear incidence rate. Motivated by [13], in this paper, we assume that there are two cases noninteracting susceptible subclasses. We denote the density of the susceptible individuals that belong to different subclasses, the infected individuals, and the recovered individuals in the population by $S_{1}, S_{2}, I$ and $R$, respectively, that is, the total variable population $N=S_{1}+S_{2}+I+R$. Moreover, if the nonlinear incidence $\beta I S^{p}(p \geq 1)$, different constant recruitments and death rates are incorporated into model (1). Then the corresponding model is investigated:

$$
\left\{\begin{array}{l}
S_{1}^{\prime}(t)=\lambda_{1}-\beta_{1} S_{1}^{p}(t) I(t)-\mu_{1} S_{1}(t), \\
S_{2}^{\prime}(t)=\lambda_{2}-\beta_{2} S_{2}^{p}(t) I(t)-\mu_{2} S_{2}(t), \\
I^{\prime}(t)=\beta_{1} S_{1}^{p}(t) I(t)+\beta_{2} S_{2}^{p}(t) I(t)-\beta_{1} e^{-d \tau} S_{1}^{p}(t-\tau) I(t-\tau)-\beta_{2} e^{-d \tau} S_{2}^{p}(t-\tau) I(t-\tau)-d I(t), \\
R^{\prime}(t)=\beta_{1} e^{-d \tau} S_{1}^{p}(t-\tau) I(t-\tau)+\beta_{2} e^{-d \tau} S_{2}^{p}(t-\tau) I(t-\tau)-\sigma R(t), \\
S_{1}\left(t^{+}\right)=\left(1-\theta_{1}\right) S_{1}(t), \\
S_{2}\left(t^{+}\right)=\left(1-\theta_{2}\right) S_{2}(t), \\
I\left(t^{+}\right)=I(t), \\
R\left(t^{+}\right)=R(t)+\theta_{1} S_{1}(t)+\theta_{2} S_{2}(t),
\end{array}\right\} \quad t=n T, n \in N, n \in N
$$

where $\lambda_{i},(i=1,2)$ are the recruitment rate into the $i$-th susceptible class, respectively parameters $\mu_{i},(i=1,2)$, $\mathrm{d}$ and $\sigma$ are the death rates of the susceptible, infected and recovered individuals, and $\beta_{i},(i=1,2)$ are the contact rates, $\tau$ is the infectious period. The term $\beta_{i} e^{-d \tau} S^{p}(t-\tau) I(t-\tau),(i=1,2)$ reflects the fact that an individual has recovered from the infective compartments and are still alive after infectious period $\tau$. $\theta_{i},(i=1,2)$ are the vaccination rates, and $T$ is the period of pulsing.

Adding all the equations in model (2), the total variable population size is given by the differential equation

$$
N^{\prime}(t)=\lambda_{1}+\lambda_{2}-\mu_{1} S_{1}(t)-\mu_{2} S_{2}(t)-d I(t)-\sigma R(t) .
$$

and we have

$$
N^{\prime}(t) \leq \lambda_{1}+\lambda_{2}-h N(t) .
$$

where $h=\min \left\{\mu_{1}, \mu_{2}, d, \sigma\right\}$. It follows that

$$
\limsup _{t \rightarrow \infty} N(t) \leq \frac{\lambda_{1}+\lambda_{2}}{h} \text {. }
$$

Note that the first three equations of system (2) do not depend on the forth equation. Thus, we restrict our attention to the following reduced system:

$$
\left\{\begin{array}{l}
S_{1}^{\prime}(t)=\lambda_{1}-\beta_{1} S_{1}^{p}(t) I(t)-\mu_{1} S_{1}(t), \\
S_{2}^{\prime}(t)=\lambda_{2}-\beta_{2} S_{2}^{p}(t) I(t)-\mu_{2} S_{2}(t), \\
I^{\prime}(t)=\beta_{1} S_{1}^{p}(t) I(t)+\beta_{2} S_{2}^{p}(t) I(t)-\beta_{1} e^{-d \tau} S_{1}^{p}(t-\tau) I(t-\tau)-\beta_{2} e^{-d \tau} S_{2}^{p}(t-\tau) I(t-\tau)-d I(t), \\
S_{1}\left(t^{+}\right)=\left(1-\theta_{1}\right) S_{1}(t), \\
S_{2}\left(t^{+}\right)=\left(1-\theta_{2}\right) S_{2}(t), \\
I\left(t^{+}\right)=I(t),
\end{array}\right\} t \neq n T, n \in N
$$

The initial conditions of (3) are

$$
S_{1}(t)=\phi_{1}(t), S_{2}(t)=\phi_{2}(t), I(t)=\phi_{3}(t) \text {, for } t \in[-\tau, 0] \text {, }
$$

where $\phi=\left(\phi_{1}, \phi_{2}, \phi_{3}\right)^{T} \in P C_{+}$, and $P C_{+}$is the space of all piecewise functions $\phi:[-\tau, 0] \rightarrow R_{+}^{3}$ with points of discontinuity at $-n T(n \in N)$ of the first kind and 
which are continuous from the left, i.e., $\phi(-n T-0)=$ $\phi(-n T)$, and $R_{+}^{3}=\left\{\left(x_{1}, x_{2}, x_{3}\right) \in R^{3} \mid x_{i} \geq 0, i=1,2,3\right\}$.

Define a subset of $R_{+}^{3}$

$$
\begin{aligned}
\Omega= & \left\{\left(S_{1}(t), S_{2}(t), I(t)\right) \in R_{+}^{3} \mid\right. \\
& \left.0 \leq S_{1}(t)+S_{2}(t)+I(t) \leq \frac{\lambda_{1}+\lambda_{2}}{h}\right\}
\end{aligned}
$$

From biological considerations, we discuss system (3) in the closed set. It is easy to verify that $\Omega$ is positively invariant with respect to system (3).

\section{Global Attractivity of Disease-Free Periodic Solution}

To prove our main results, we state some notations and lemmas which will be essential to our proofs.

Lemma 1 (see [6]) Consider the following impulsive differential equation

$$
\left\{\begin{array}{l}
u^{\prime}(t)=a-b u(t), t \neq n T, \\
u\left(t^{+}\right)=(1-\theta) u(t), t=n T,
\end{array}\right.
$$

where $a>0, b>0,0<\theta<1$. Then above system exists $a$ unique positive periodic solution given by

$$
u^{*}(t)=\frac{a}{b}+\left(\bar{u}-\frac{a}{b}\right) e^{-b(t-n T)}, \text { for } n T<t \leq(n+1) T,
$$

which is globally asymptotically stable, where

$$
\bar{u}=\frac{a}{b} \frac{(1-\theta)\left(1-e^{-b T}\right)}{1-(1-\theta) e^{-b T}} .
$$

Definition 1 (see [14]). Let $V: R_{+} \times R_{+}^{3} \rightarrow R_{+}$, then $V$ is said to belong to class $V_{0}$ if

i) $V$ is continuous in $(n T,(n+1) T] \times R_{+}^{3}$ and for each $X \in R_{+}^{3}, \quad \lim _{(t, y) \rightarrow\left(n T^{+}, X\right)} V(t, y)=V\left(n T^{+}, X\right)$ exists.

ii) $V$ is locally Lipschitzian in $X$.

Lemma 2 (see [14]). Let $V \in V_{0}$. Assume that

$$
\begin{cases}D^{+} V(t, x) \leq g(t, V(t, x)), & t \neq n T, \\ V\left(t, x\left(t^{+}\right)\right) \leq \varphi_{n}(V(t, x)), & t=n T,\end{cases}
$$

where $g: R_{+} \times R_{+} \rightarrow R$ is continuous in $(n T,(n+1) T] \times R_{+}$ and for $u \in R_{+}, \quad n \in N, \lim _{(t, y) \rightarrow\left(n T^{+}, u\right)} V(t, y)=V\left(n T^{+}, u\right)$ exists, $\varphi_{n}: R_{+} \rightarrow R_{+}$is non-decreasing. Let $r(t)$ be the maximal solution of the scalar impulsive differential equation

$$
\begin{cases}u^{\prime}(t)=g(t, u(t)), & t \neq n T, \\ u\left(t^{+}\right)=\varphi_{n}(u(t)), & t=n T, \\ u\left(0^{+}\right)=u_{0}, & \end{cases}
$$

existing on $[0, \infty]$. Then $V\left(0^{+}, x_{0}\right) \leq u_{0}$ implies that $V(t, x(t)) \leq r(t), t \geq 0$, where $x(t)$ is any solution of (3).

In the following we shall demonstrate that the disease-free periodic solution $\left(S_{1}^{*}(t), S_{2}^{*}(t), 0\right)$ is global attractive. We firstly show the existence of the disease-free periodic solution, in which the infectious individuals are entirely absent from the population permanently, i.e. $I(t) \equiv 0$ for all $t>0$. Under this condition, the growth of the $i$-th $(i=1,2)$ susceptible individuals must satisfy

$$
\left\{\begin{array}{l}
S_{i}^{\prime}(t)=\lambda_{i}-\mu_{i} S_{i}(t), \quad t \neq n T, \\
S_{i}\left(t^{+}\right)=(1-\theta) S_{i}(t), \quad t=n T .
\end{array}\right.
$$

According to Lemma 1, we know that the periodic solution of the system

$$
\begin{aligned}
& S_{i}^{*}(t)=\frac{\lambda_{i}}{\mu_{i}}\left(1-\frac{\theta_{i} e^{-\mu_{i}(t-n T)}}{1-\left(1-\theta_{i}\right) e^{-\mu_{i} T}}\right) \\
& \text { for } n T<t \leq(n+1) T, \quad(i=1,2)
\end{aligned}
$$

is globally asymptotically stable. Therefore system (3) has a unique disease-free periodic solution

$$
\left(S_{1}^{*}(t), S_{2}^{*}(t), 0\right) \text {. }
$$

Denote

$$
R_{1}=\frac{\beta_{1}\left(\frac{\lambda_{1}}{\mu_{1}} \frac{1-e^{-\mu_{1} T}}{1-\left(1-\theta_{i}\right) e^{-\mu_{1} T}}\right)^{p}+\beta_{2}\left(\frac{\lambda_{2}}{\mu_{2}} \frac{1-e^{-\mu_{2} T}}{1-\left(1-\theta_{2}\right) e^{-\mu_{2} T}}\right)^{p}}{d} .
$$

Theorem 1 If $R_{1}<1$, then the disease-free periodic solution $\left(S_{1}^{*}(t), S_{2}^{*}(t), 0\right)$ of system (3) is globally attractive.

Proof. Since $R_{1}<1$, we can choose $\varepsilon>0$ sufficiently small such that

$$
\begin{aligned}
& \beta_{1}\left(\frac{\lambda_{1}}{\mu_{1}} \frac{1-e^{-\mu_{1} T}}{1-\left(1-\theta_{i}\right) e^{-\mu_{1} T}}+\varepsilon\right)^{p} \\
& +\beta_{2}\left(\frac{\lambda_{2}}{\mu_{2}} \frac{1-e^{-\mu_{2} T}}{1-\left(1-\theta_{2}\right) e^{-\mu_{2} T}}+\varepsilon\right)^{p}<d .
\end{aligned}
$$

From the first equation and the second equation of system (3), we have $S_{i}^{\prime}(t)<\lambda_{i}-\mu_{i} S_{i}(t), \quad(i=1,2)$. Then we consider the following impulsive comparison system 


$$
\left\{\begin{array}{l}
u_{i}^{\prime}(t)=\lambda_{i}-\mu_{i} u_{i}(t), t \neq n T, \\
u_{i}\left(t^{+}\right)=(1-\theta) u_{i}(t), t=n T,
\end{array}\right.
$$

According to Lemma 1, we obtain the periodic solution of system (7)

$$
\begin{aligned}
& u_{i}^{*}(t)=\frac{\lambda_{i}}{\mu_{i}}\left(1-\frac{\theta_{i} e^{-\mu_{i}(t-n T)}}{1-\left(1-\theta_{i}\right) e^{-\mu_{i} T}}\right), \\
& \text { for } n T<t \leq(n+1) T, \quad(i=1,2),
\end{aligned}
$$

which is globally asymptotically stable. By the comparison theorem [14], we have that there exists $n_{1} \in Z_{+}$ such that for $n T<t \leq(n+1) T, n>n_{1}$

$$
\begin{aligned}
S_{i}(t)< & u_{i}^{*}(t)+\varepsilon \leq \frac{\lambda_{i}}{\mu_{i}}\left(1-\frac{\theta_{i} e^{-\mu_{i} T}}{1-\left(1-\theta_{i}\right) e^{-\mu_{i} T}}\right) \\
& +\varepsilon=\bar{S}_{i}, \quad(i=1,2) .
\end{aligned}
$$

Furthermore, from the third equation of system (3), we get $I^{\prime}(t)<\left(\beta_{1} \bar{S}_{1}^{p}+\beta_{2} \bar{S}_{2}^{p}-d\right) I(t)$ for $t>n T$ and $n>n_{1}$.

From (6) and (8), we have $I^{\prime}(t)<0$, then $\lim _{t \rightarrow \infty} I(t)=0$, i.e., for any sufficiently small $\varepsilon_{1}>0$, there exists an integer $n_{2}>n_{1}$ such that $I(t)<\varepsilon_{1}$, for all $t>n_{2} T$.

From the first equation and the second equation of system (3), we have for $t>n_{2} T$

$$
S_{i}^{\prime}(t)>\lambda_{i}-\left(\beta_{i} \bar{S}_{i}^{p-1} \varepsilon_{1}+\mu_{i}\right) S_{i}(t), \quad(i=1,2) .
$$

Then we consider the following impulsive comparison systems

$$
\left\{\begin{array}{l}
v_{i}^{\prime}(t)=\lambda_{i}-\left(\beta_{i} \bar{S}_{i}^{p-1} \varepsilon_{1}+\mu_{i}\right) v_{i}(t), t \neq n T, \\
v_{i}\left(t^{+}\right)=\left(1-\theta_{i}\right) v_{i}(t), t=n T
\end{array}\right.
$$

From Lemma 1, we obtain the periodic solution of system (9)

$$
v_{i}^{*}(t)=\frac{\lambda_{i}}{\beta_{i} \bar{S}_{i}^{p-1} \varepsilon_{1}+\mu_{i}}\left(1-\frac{\theta_{i} e^{-\left(\beta_{i} \bar{S}_{i}^{p-1} \varepsilon_{1}+\mu_{i}\right)(t-n T)}}{1-\left(1-\theta_{i}\right) e^{-\left(\beta_{i} \bar{S}_{i}^{p-1} \varepsilon_{1}+\mu_{i}\right) T}}\right), \quad n T<t \leq(n+1) T, \quad(i=1,2),
$$

which is globally asymptotically stable. In view of the comparison theorem [14], there exists an integer $n_{3}>n_{2}$

such that for $n T<t \leq(n+1) T, \quad n>n_{3}$,

$$
S_{i}(t)>v_{i}^{*}(t)+\varepsilon \geq \frac{\lambda_{i}}{\beta_{i} \bar{S}_{i}^{p-1} \varepsilon_{1}+\mu_{i}}\left(1-\frac{\theta_{i} e^{-\left(\beta_{i} \bar{S}_{i}^{p-1} \varepsilon_{1}+\mu_{i}\right) T}}{1-\left(1-\theta_{i}\right) e^{-\left(\beta_{i} \bar{S}_{i}^{p-1} \varepsilon_{1}+\mu_{i}\right) T}}\right)+\varepsilon,(i=1,2)
$$

Since $\varepsilon$ and $\varepsilon_{1}$ are sufficiently small, from (8) and (10), we know that

$$
\lim _{t \rightarrow \infty} S_{i}(t)=S^{*}(t), \quad(i=1,2) .
$$

Hence, disease-free periodic solution $\left(S_{1}^{*}(t), S_{2}^{*}(t), 0\right)$ of system (3) is globally attractive. The proof is completed.

Next, we give some accounts of the Theorem 1 for a well biological meaning.

$$
\frac{\partial R_{1}}{\partial T}=p \frac{\beta_{1}}{d} \frac{\lambda_{1}^{p}}{\mu_{1}^{p}} \frac{\left(1-e^{-\mu_{1} T}\right)^{p-1}}{\left(1-\left(1-\theta_{1}\right) e^{-\mu_{1} T}\right)^{p+1}} \theta_{1} \mu_{1} e^{-\mu_{1} T}+p \frac{\beta_{2}}{d} \frac{\lambda_{2}^{p}}{\mu_{2}^{p}} \frac{\left(1-e^{-\mu_{2} T}\right)^{p-1}}{\left(1-\left(1-\theta_{2}\right) e^{-\mu_{2} T}\right)^{p+1}} \theta_{2} \mu_{2} e^{-\mu_{2} T}>0 .
$$

Theorem 1 determines the global attravtivity of the disease-free periodic solution of system (3) in $\Omega$ for the case $R_{1}<1$. Its epidemiology implies that the disease will die out. From (11) and (12), we can see that larger pulse vaccination rates or a shorter period of immune vaccination will make for the disease eradication.

\section{Permanence}

In this section, we state the disease is endemic if the in-
By simple calculation, from (5) we get

$$
\begin{aligned}
& \frac{\partial R_{1}}{\partial \theta_{1}}=-p \frac{\beta_{1}}{d} \frac{\lambda_{1}^{p}}{\mu_{1}^{p}} \frac{\left(1-e^{-\mu_{1} T}\right)^{p}}{\left(1-\left(1-\theta_{1}\right) e^{-\mu_{1} T}\right)^{p+1}} e^{-\mu_{1} T}<0, \\
& \frac{\partial R_{1}}{\partial \theta_{2}}=-p \frac{\beta_{2}}{d} \frac{\lambda_{2}^{p}}{\mu_{2}^{p}} \frac{\left(1-e^{-\mu_{2} T}\right)^{p}}{\left(1-\left(1-\theta_{2}\right) e^{-\mu_{2} T}\right)^{p+1}} e^{-\mu_{2} T}<0,
\end{aligned}
$$

fectious population persists above a certain positive level for sufficiently large time. The endemicity of the disease can be well captured and studied through the notation of uniform persistence.

Definition 2. System (3) is said to be uniformly persistent if there exist positive constants $M_{i} \geq m_{i}>0$ $(i=1,2,3)$ (both are independent of the initial values), such that every solution $\left(S_{1}(t), S_{2}(t), I(t)\right)$ with positive initial conditions of system (3) satisfies

$$
m_{1} \leq S_{1}(t) \leq M_{1}, m_{2} \leq S_{2}(t) \leq M_{2}, m_{3} \leq I(t) \leq M_{3} .
$$


Denote

$$
R_{2}=\frac{\beta_{1}\left(\frac{\lambda_{1}}{\mu_{1}} \frac{1-e^{-\mu_{1} T}}{1-\left(1-\theta_{1}\right) e^{-\mu_{1} T}}\right)^{p}\left(1-e^{-d \tau}\right)+\beta_{2}\left(\frac{\lambda_{2}}{\mu_{2}} \frac{1-e^{-\mu_{2} T}}{1-\left(1-\theta_{2}\right) e^{-\mu_{2} T}}\right)^{p}\left(1-e^{-d \tau}\right)}{d} .
$$

Theorem 2. If $R_{2}>1$, then system (3) is uniformly persistent.

$$
S_{1}(t) \leq \frac{\lambda_{1}+\lambda_{2}}{h}, \quad S_{2}(t) \leq \frac{\lambda_{1}+\lambda_{2}}{h}, \quad I(t) \leq \frac{\lambda_{1}+\lambda_{2}}{h}, \quad \text { for all } t>0 .
$$

We are left to prove there exist positive constants $m_{1}, m_{2}, m_{3}$ such that $S_{1}(t) \geq m_{1}, S_{2}(t) \geq m_{2}, I(t) \geq m_{3}$, for all sufficiently large $t$.

Firstly, from the first and second equations of system (3), we have

$$
S_{i}^{\prime}(t) \geq \lambda_{i}-\left(\beta_{i}\left(\frac{\lambda_{1}+\lambda_{2}}{h}\right)^{p}+\mu_{i}\right) S_{i}(t),(i=1,2) .
$$

Considering the following comparison equations

$$
\begin{aligned}
& S_{i}(t) \geq u_{i}(t) \geq u_{i}^{*}(t)-\tilde{\varepsilon} \geq \frac{\lambda_{i}}{\beta_{i}\left(\frac{\lambda_{1}+\lambda_{2}}{h}\right)^{p}+\mu_{i}}\left(1-\frac{\theta_{i} e^{-\left(\beta_{i}\left(\frac{\lambda_{1}+\lambda_{2}}{h}\right)^{p}+\mu_{i}\right) T}}{1-(1-\theta)_{i} e^{-\left(\beta_{i}\left(\frac{\lambda_{1}+\lambda_{2}}{h}\right)^{p}+\mu_{i}\right) T}}\right)-\tilde{\varepsilon}=m_{i} . \\
& \beta_{1} \eta_{1}^{p}\left(1-e^{-d \tau}\right)+\beta_{2} \eta_{2}^{p}\left(1-e^{-d \tau}\right)>d,
\end{aligned}
$$

Now, we shall prove there exist a $m_{3}>0$ such that $I(t) \geq m_{3}$ for all sufficiently large $t$. For convenience, we prove it through the following two steps:

Step I. Since $R_{2}>1$, there exist sufficiently small

where

$$
\eta_{i}=\frac{\lambda_{i}}{\beta_{i}\left(\frac{\lambda_{1}+\lambda_{2}}{h}\right)^{p} m_{I}^{*}+\mu_{i}}\left(1-\frac{\theta_{i} e^{-\left(\beta_{i}\left(\frac{\lambda_{1}+\lambda_{2}}{h}\right)^{p} m_{I}^{*}+\mu_{i}\right) T}}{1-\left(1-\theta_{i}\right) e^{-\left(\beta_{i}\left(\frac{\lambda_{1}+\lambda_{2}}{h}\right)^{p} m_{I}^{*}+\mu_{i}\right) T}}\right)-\bar{\varepsilon}, \quad(i=1,2) .
$$

For $m_{I}^{*}$, we can claim that there exists a $t_{1}>0$ such that $I\left(t_{1}\right) \geq m_{I}^{*}$. Otherwise, $I(t)<m_{I}^{*}$ for all $t>0$. It follows from the first and second equations of (3) that

$$
S_{i}^{\prime}(t) \geq \lambda_{i}-\left(\beta_{i}\left(\frac{\lambda_{1}+\lambda_{2}}{h}\right)^{p-1} m_{I}^{*}+\mu_{i}\right) S_{i}(t),(i=1,2) .
$$

Considering the following impulsive comparison sys-

$$
\left\{\begin{array}{l}
v_{i}^{\prime}(t)=\lambda_{i}-\left(\beta_{i}\left(\frac{\lambda_{1}+\lambda_{2}}{h}\right)^{p-1} m_{I}^{*}+\mu_{i}\right) v_{i}(t), t \neq n T \\
v_{i}\left(t^{+}\right)=\left(1-\theta_{i}\right) v_{i}(t), t=n T
\end{array}\right.
$$

Similarly, we know that there exists $t_{2}>0$, such that tems

$$
S_{i}(t) \geq v_{i}(t) \geq v_{i}^{*}(t)-\tilde{\varepsilon} \geq \frac{\lambda_{i}}{\beta_{i}\left(\frac{\lambda_{1}+\lambda_{2}}{h}\right)^{p-1} m_{I}^{*}+\mu_{i}}\left(1-\frac{\theta_{i} e^{-\left(\beta_{i}\left(\frac{\lambda_{1}+\lambda_{2}}{h}\right)^{p-1} m_{I}^{*}+\mu_{i}\right)^{T}}}{1-\left(1-\theta_{i}\right) e^{-\left(\beta_{i}\left(\frac{\lambda_{1}+\lambda_{2}}{h}\right)^{p-1} m_{I}^{*}+\mu_{i}\right) T}}\right)-\bar{\varepsilon}=\eta_{i} .
$$

Further, the third equations of system (3) can be rewritten as

$$
\begin{gathered}
I^{\prime}(t)=\beta_{1} S_{1}^{p}(t) I(t)+\beta_{2} S_{2}^{p}(t) I(t) \\
-\beta_{1} e^{-d \tau} S_{1}^{p}(t-\tau) I(t-\tau)
\end{gathered}
$$

$$
\begin{aligned}
& -\beta_{2} e^{-d \tau} S_{2}^{p}(t-\tau) I(t-\tau)-d I(t) \\
= & \beta_{1} S_{1}^{p}(t) I(t)\left(1-e^{-d \tau}\right)+\beta_{2} S_{2}^{p}(t) I(t)\left(1-e^{-d \tau}\right) \\
& -d I(t)+\beta_{1} e^{-d \tau} \frac{d}{d t} \int_{t-\tau}^{t} S_{1}^{p}(\xi) I(\xi) d \xi
\end{aligned}
$$




$$
+\beta_{2} e^{-d \tau} \frac{d}{d t} \int_{t-\tau}^{t} S_{2}^{p}(\xi) I(\xi) d \xi
$$

Define

$$
\begin{aligned}
V(t)= & I(t)-\beta_{1} e^{-d \tau} \int_{t-\tau}^{t} S_{1}^{p}(\xi) I(\xi) d \xi \\
& -\beta_{2} e^{-d \tau} \int_{t-\tau}^{t} S_{2}^{p}(\xi) I(\xi) d \xi .
\end{aligned}
$$

For $t>t_{2}$, the derivative of $V(t)$ along the solution of system (3) is

$$
\begin{aligned}
V^{\prime}(t) & =\left[\beta_{1} S_{1}^{p}(t)\left(1-e^{-d \tau}\right)+\beta_{2} S_{2}^{p}(t)\left(1-e^{-d \tau}\right)-d\right] I(t) \\
> & {\left[\beta_{1} \eta_{1}^{p}\left(1-e^{-d \tau}\right)+\beta_{2} \eta_{2}^{p}\left(1-e^{-d \tau}\right)-d\right] I(t) }
\end{aligned}
$$

From (13), we have $V^{\prime}(t)>0$ for $t>t_{2}$, which implies that $V(t) \rightarrow \infty$ as $t \rightarrow \infty$. This contradicts the boundedness of $V(t)$. Hence, there exists a $t_{1}>0$ such that $I\left(t_{1}\right) \geq m_{I}^{*}$.

Step II. According to step I, for any positive solution $\left(S_{1}(t), S_{2}(t), I(t)\right)$ of (3), we are left to consider two cases. First, If $I(t)>m_{I}^{*}$ for all $t>t_{1}$, then our result is proved. Second, if $I(t)<m_{I}^{*}$ for some $t>t_{1}$, we can choose constants $\rho>0$ and $T_{0}>\max \left\{t_{2}, t_{1}+\tau\right\} \quad\left(T_{0}\right.$ is sufficiently large) such that $I(t) \leq m_{I}^{*}, I\left(T_{0}\right)=m_{I}^{*}$, $I\left(T_{0}+\rho\right)=m_{I}^{*} \quad$ and $\quad S_{1}(t)>\eta_{1}, S_{2}(t)>\eta_{2} \quad$ for $t \in\left[T_{0}, T_{0}+\rho\right]$.

Thus, there exists a $g(0<g<\tau)$ such that for $t \in\left[T_{0}, T_{0}+g\right]$

$$
I(t) \geq \frac{m_{I}^{*}}{2} .
$$

In this case, we shall discuss three possible cases in term of the sizes of $g, \rho$ and $\tau$.

Case 1. If $\rho \leq g<\tau$, then it is obvious that $I(t) \geq m_{I}^{*} / 2$, for $t \in\left[T_{0}, T_{0}+\rho\right]$.

Case 2. If $g<\rho \leq \tau$, then from the third equation of (3), we can deduce

$$
I(t)=\int_{t-\tau}^{t}\left(\beta_{1} S_{1}^{p}(\xi)+\beta_{2} S_{2}^{p}(\xi)\right) I(\xi) e^{-d(t-\xi)} d \xi,
$$

From (14), (15) and (16), we have

$$
\begin{aligned}
I(t) & >\int_{T_{0}}^{T_{0}+g}\left(\beta_{1} S_{1}^{p}(\xi)+\beta_{2} S_{2}^{p}(\xi)\right) I(\xi) e^{-d(t-\xi)} d \xi, \text { for } t \in\left[T_{0}+g, T_{0}+\rho\right] \\
& >\left(\beta_{1} \eta_{1}^{p}+\beta_{2} \eta_{2}^{p}\right) \frac{m_{I}^{*}}{2} e^{-d \tau} g=m_{I}^{* * *}
\end{aligned}
$$

Set $m_{3}=\min \left\{\frac{m_{I}^{*}}{2}, m_{I}^{* *}\right\}>0$, for $t \in\left[T_{0}, T_{0}+\rho\right]$, we have $I(t)>m_{3}$.

Case 3. If $g<\tau \leq \rho$, we will discuss the following two cases, respectively.
Case 3.1. For $t \in\left[T_{0}, T_{0}+\tau\right]$, it is easy to obtain $I(t)>m_{I}^{* *}$.

Case 3.2. For $t \in\left[T_{0}+\tau, T_{0}+\rho\right]$. We claim that $I(t)>m_{I}^{* * *}$. Otherwise, there exists a $\bar{t} \in\left[T_{0}+\tau, T_{0}+\rho\right]$, such that $I(t)>m_{I}^{* *}$ for $t \in\left[T_{0}+\tau, \bar{t}\right]$, and $I(\bar{t})=m_{I}^{* *}$. From (13), (14) and (16), we have

$$
I(\bar{t})=\int_{\bar{t}-\tau}^{\bar{t}}\left(\beta_{1} S_{1}^{p}(\xi)+\beta_{2} S_{2}^{p}(\xi)\right) I(\xi) e^{-d(\bar{t}-\xi)} d \xi>\left(\beta_{1} \eta_{1}^{p}+\beta_{2} \eta_{2}^{p}\right) m_{I}^{* *} \frac{1-e^{-d \tau}}{d}>m_{I}^{* *},
$$

which is contradictory to $I(\bar{t})=m_{I}^{* *}$. Hence, the claim holds true.

According to the arbitrary of $T_{0}$, we can obtain that $I(t)>m_{3}$ holds for all $t>T_{0}$. The proof is completed.

\section{Numerical Simulations}

In this section, we give some numerical simulations to illustrate the effects of different probability on population. In system (3), $\lambda_{1}=0.25, \quad \lambda_{2}=0.2, \quad \beta_{1}=0.03$, $\beta_{2}=0.1, \quad \mu_{1}=0.03, \quad \mu_{2}=0.05, \quad p=2, \quad d=0.1, \quad \tau=2$, $T=3$. Time series are drawn in Figure 1(a) and Figure 1(b) with initial values $\phi_{1}(t)=2+0.5 \sin t, \quad \phi_{2}(t)=$ $2+0.8 \cos t, \phi_{3}(t)=1+0.5 \sin t, t \in[-2,0]$ for 30 pulsing cycles. If we take $\theta_{1}=0.45, \theta_{2}=0.90$, then $R_{1}=$ 0.9956 . By Theorem 1, we know that the disease will disappear (see Figure 1(a)). If we let $\theta_{1}=0.10, \quad \theta_{2}=$
0.20 , then $R_{2}=1.4685$. According to Theorem 2, we know that the disease will be permanent (see Figure 1(b)).

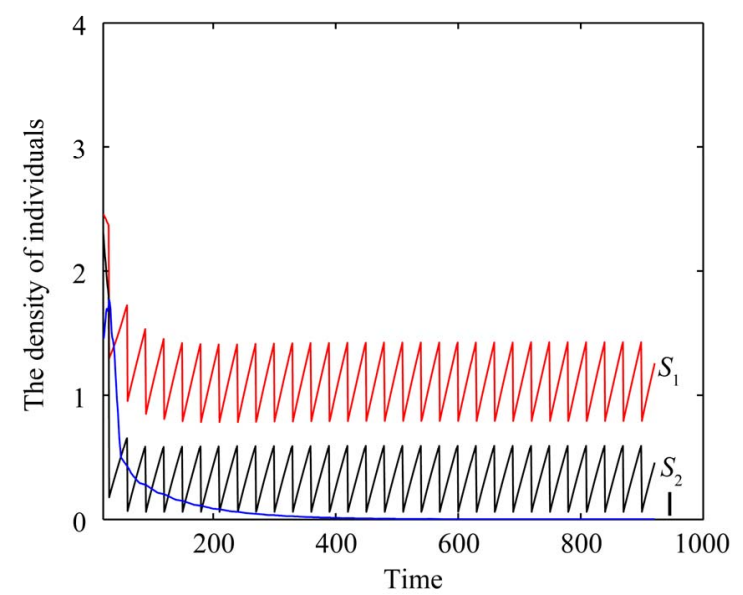

(a) 


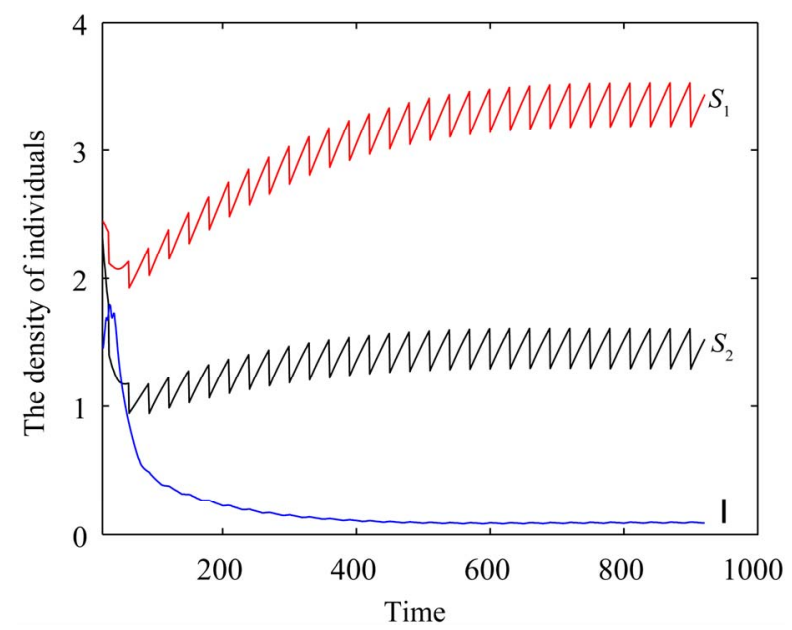

(b)

Figure 1. Two figures show that movement paths of $S_{1}, S_{2}$ and $I$ as functions of time $t$. (a) Disease will be extinct with $R_{1}=0.9956$ and $\theta_{1}=0.45, \theta_{2}=0.9$; (b) Disease will be persistent with $R_{2}=1.4685$ and $\theta_{1}=0.1, \theta_{2}=0.2$.

\section{Acknowledgements}

The research of Shujing Gao has been supported by The Natural Science Foundation of China (10971037) and The National Key Technologies R \& D Program of China (2008BAI68B01).

\section{References}

[1] X. B. Zhang, H. F. Huo, X. K. Sun and Q. Fu, "The Differential Susceptibility SIR Epidemic Model with Time Delay and Pulse Vaccination,” Journal of Applied Mathematics and Computing, Vol. 34, No. 1-2, 2009, pp. 287298.

[2] Z. Agur, L. Cojocaru, R. Anderson and Y. Danon, "Pulse Mass Measles Vaccination across Age Cohorts," Proceedings of the National Academy of Sciences of the United States of America, Vol. 90, No. 24, 1993, pp. 11698-11702. doi:10.1073/pnas.90.24.11698

[3] W. O. Kermack and A. G. McKendrick, “Contributions to the Mathematical Theory of Epidemics-II: The Problem of Endemicity,” Proceedings of the Royal Society Series A, Vol. 138, No. 834, 1932, pp. 55-83. doi:10.1098/rspa. 1932.0171

[4] W. O. Kermack and A. G. McKendrick, "Contributions to the Mathematical Theory of Epidemics-III: Further Studies of the Problem of Endemicity," Proceedings of the Royal Society Series A, Vol. 141, No. 843, 1933, pp. 94-122. doi:10.1098/rspa.1933.0106

[5] R. M. Anderson and R. M. May, "Population Biology of Infectious Disease: Part I,” Nature, Vol. 280, 1979, pp. 361-367. doi:10.1038/280361a0

[6] S. Gao, L. Chen and J. J. Nieto, “Angela Torres, Analysis of a Delayed Epidemic Model with Pulse Vaccination and Saturation Incidence,” Vaccine, Vol. 24, No. 35-36, 2006, pp. 6037-6045. doi:10.1016/j.vaccine.2006.05.018

[7] C. McCluskey, "Global Stability for a Class of Mass Action Systems Allowing for Latency in Tuberculosis," Journal of Mathematical Analysis and Applications, Vol. 338, No. 1, 2008, pp. 518-535. doi:10.1016/j.jmaa.2007. 05.012

[8] K. L. Cooke and P. van den Driessche, "Analysis of an SEIRS Epidemic Model with Two Delays,” Journal of Mathematical Biology, Vol. 35, No. 2, 1996, pp. 240-260. doi:10.1007/s002850050051

[9] R. M. Anderson and R. M. May, "Infectious Diseases of Humans, Dynamics and Control,” Oxford University Press, Oxford, 1992.

[10] O. Diekmann and J. A. P. Heesterbeek, "Mathematical Epidemiology of Infectious Diseases,” John Wiley \& Sons, Chisteter, 2000.

[11] F. Brauer and C. C. Castillo, "Mathematical Models in Population Biology and Epidemiology,” Springer, New York, 2000.

[12] W. O. Kermack and A. G. McKendrick, “A Contribution to the Mathematical Theory of Epidemics I,” Proceedings of the Royal Society Series A, Vol. 115, No. 772, 1927, pp. 700-721. doi:10.1098/rspa.1927.0118

[13] S. Gao, Z. Teng and D. Xie, "Analysis of a Delayed SIR Epidemic Model with Pulse Vaccination," Chaos, Solitons \& Fractals, Vol. 40, No. 2, 2009, pp. 1004-1011. doi:10.1016/j.chaos.2007.08.056

[14] V. Lakshmikantham, D. D. Bainov and P. S. Simeonov, "Theory of Impulsive Differential Equations," World Scientific, Singapore, 1989. 